\title{
Mapping organism expression levels at cellular resolution in developing Drosophila
}

\author{
David W. Knowles*, Soile Keränen+, Mark D. Biggin+, Damir Sudar* \\ Departments of Cell and Molecular Biology* and Genome Sciences+, Life Sciences Division, \\ Lawrence Berkeley National Laboratory, Berkeley, California 94720
}

To understand transcriptional networks, we are developing novel quantitative optical imaging techniques to map gene expression at cellular resolution within an entire organism. Pregastrula Drosophila embryos have been chosen because they allow high resolution 3D optical imaging and the transcription network controlling gene expression in early Drosophila is well characterized. Embryos at different stages of development are labeled for total DNA and specific gene products using different fluorophors and imaged in 3D with confocal microscopy. Novel algorithms have been developed which allow the DNA-image to be automatically segmented to produce a morphological mask of the individual nuclear boundaries. The morphological mask is initially used to count the total number of nuclei with an embryos. We find that the total nuclear number clearly defines the mitotic division number and use this to stage individual embryo. The morphological mask is then used to quantify gene-product on a per nucleus basis. By studying changes in the patterns of specific gene products with developmental stage, we are uncovering the transcriptional regulatory network in early Drosophila. 

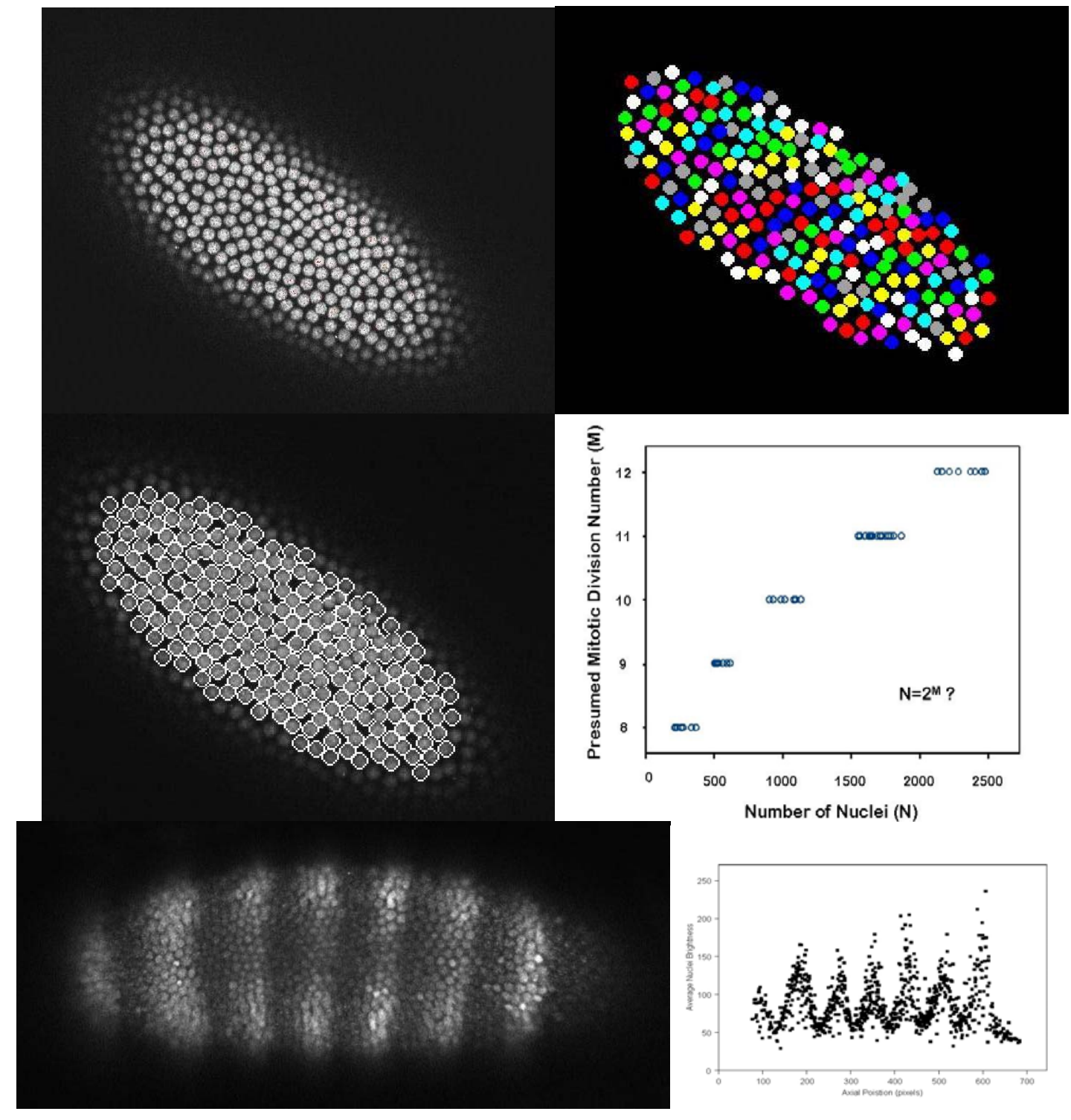

Figure 1: To calculate the position and extent of individual nuclear volumes, a local threshold algorithm analyzes the DNA-stain image (Top Left) and a template matching algorithm stamps a non-overlapping, enumerated template of approximate nuclear volume centered on the nuclei (Top Right). The accuracy of the segmentation is demonstrated by overlaying an outline of the segmentation mask over the original gray level image (Middle Left). In each case only a single slice from a 3D image is shown. For the plot of mitotic division number versus total nuclei number, images of 55 embryo at various growth stages were segmented and analyzed for total nuclear number. The results showed that individual embryo could be grouped according to total nuclear number. Thus, we have plotted the total number of nuclei (N) (abscissa), from the 55 embryos, against the presumed mitotic division number (M) (ordinate). $\mathrm{M}$ was calculated from the relation $\mathrm{N}=2 \mathrm{M}$ and by assuming that $\mathrm{N}$ follows this power law for at least the first 8 mitotic divisions. For enumeration purposes, the accuracy was nearly $100 \%$. Finally, by analyzing the gene-product image of Texas-Red stained EVE protein (Bottom Left) we have calculated the relative amount of EVE expression per nuclei within an entire embryo. The relative amount of EVE expression from this image is plotted against the average relative anterior/posterior position of the nuclei within the embryo (Bottom Right). 